\title{
Cognitive Ad Hoc Network Dynamic Spectrum Access Channel Strategy
}

\author{
Zhipeng Song \\ QingDao Hismile College \\ sony1787@126.com
}

\begin{abstract}
This article is based on the existing dynamic channel access strategy, proposes a cognitive MAC layer channel access strategy. First of all, from the physical layer of cognitive radio spectrum detection technology to detect the idle spectrum, provide cognitive users can access channel; Second, in the network, their cognitive users maintain a realtime update list of channel state, this strategy according to channel state list to find two cognitive users need communication overlapping channels are available, and calculate the overlapping each sum of the available channel utilization rate of the primary user; Finally choose the utilization ratio of the sum of the minimum channel for data transmission, improve the data channel available transfer time. The simulation results show that the strategy under the condition of without affecting the main user communication can make the cognitive users to make full use of the authorized spectrum, improve the network throughput.
\end{abstract}

Keywords: Cognitive Ad hoc network, dynamic spectrum access, spectrum detection

\section{Introduction}

In recent years, wireless mobile communication technology in broadband communication, seamless communication and intelligent communication as a benchmark and goals, the corresponding such as zigbee (WPAN), wireless wide area network (WWAN) and wireless local area network (WLAN), such as the network is in constant reform and innovation. However, with the rapid development of information society economy, the pursuit of the number of mobile communications and broadband wireless access business growing, only scarce radio spectrum resources under the trend of rapid growth of the demand, is becoming more valuable. According to statistics, in countries around the world have been allocated spectrum, more than $70 \%$ have not been fully utilized. In June 2010, the Obama administration have signed a memorandum, in the next 10 years, the government and commercial wireless spectrum auction $500 \mathrm{MHZ}$, so as to meet the increasing demand for wireless Internet connection. In 2011, the federal communications commission (FCC) and the U.S. department of commerce coordination allocate some unused spectrum resources, and proposed to $115 \mathrm{MHZ}$ spectrum allocated to wireless broadband operators, $120 \mathrm{MHZ}$ spectrum for wireless broadband communications. In China, the distribution of the radio spectrum resources used single mode of administrative examination and approval, spectrum up cost is low. The occupied spectrum has two kinds of typical state, is a free occasionally been used for a long time, serious waste; Second, with the rapid growth of the types and business users, business and crowded. It is clear that did not reflect the value of spectrum resources. At the end of 2011, administration of industry and information technology, radio frequency resources management aspect, has completed the td-scdma LTE test spectrum allocation work in planning, conducting the IMT system and Internet related frequency spectrum planning research work, formed the IMT frequency $2500 \mathrm{MHZ}$ to $2690 \mathrm{MHZ}$ frequency planning plan. 
Throughout the United States and China over the past few years the spectrum allocation and utilization of resources, obviously, only focus on developing some unused spectrum resources is not enough. As a result, most of the research is mainly on how to improve the existing available spectrum on the utilization of resources, on the one hand, orthogonal frequency division multiplexing (OFDM) technique [1], the link adaptive technology, advanced modulation coding techniques and multiple input multiple output (MIMO) technology [2,3], and so on, these methods are focused on mining available spectrum resource potential, the flow of information transmission spectrum of a unit as far as possible to improve, but did not fundamentally solve the spectrum tense situation; , on the other hand, such as medical, scientific research and industry, science and technology in areas such as frequency of open access, and has a narrow band system and uwb systems with the combination of technology, the traditional fixed spectrum sharing technology, can to a certain extent, improve the utilization rate of limited spectrum, but at the same time can significantly increase the harmful interference in the network, and sharing of this kind of fixed frequency band, low transmission power is often restricted to the short distance communication, communication system capacity and flexibility is not so good. So, it is necessary to study spectrum sharing technology is suitable for the modern communication network. In 1999 put forward by Mitola Cognitive Radio (Cognitive Radio, CR) [4, 5], from a new Angle is presented a method of effective utilization of the current spectrum. It in order to improve the spectrum utilization efficiency as the goal, automatic perception of time domain, frequency domain and spatial domain in $3 \mathrm{~d}$ space, such as wireless communication environment, adjust the operation for adaptive rf excitation statistical change, at the same time, also improved the stability of the communication system. This is a way of implementing open spectrum sharing flexibility and effectiveness, but also on the way of dynamic spectrum access are put forward higher requirements.

As Dynamic Spectrum Access (Dynamic Spectrum Access, DSA) technology has been approved for wireless TV system of free frequencies [6,7], there is no doubt that took the radio communication is an important step in the Spectrum Access Shared, also means that the radio Spectrum Access Shared ways are no longer limited to different frequency band itself, can also according to the Spectrum in the free time and space differences, according to the actual situation to dynamically Access to use, thus effectively reducing the Spectrum scarcity and bandwidth limits on the constraints of radio technology development.

\section{Related Works}

There are many kinds of spectrum detection method of cognitive radio, and these methods in computational complexity and the ability of detecting signal. As a result, the algorithm choice depends on the testing requirements and available computing resources. Spectrum detection performance usually in detection probability and the perception of time necessary to achieve target detection probability as a symbol.

Early classical energy detection method, do not need to test the main users of information, but in the low SNR region or used to detect the spread spectrum signal, it is difficult to achieve better performance; Under the condition of static Gaussian noise detection method is best matched filter detection, it needs to know the complete information of detected signal in advance, to maximize the receive SNR, but it is difficult to achieve synchronization in the low SNR region; In the region of the low SNR, the detection probability of cycle stationary test is better than energy detection, but the relatively high computational complexity and need to be better than energy detection long time perception; As long as the authorized users use authorized spectrum communication, this method can detect the user at the receiver end of the seismic signal, to authorized users. In order to improve the ability of detecting radio 
spectrum hole, will start the related researcher's attention from pure physical detection algorithm, transferred to the cross layer design scheme of MAC layer in listening. This kind of combined listening scheme, on the one hand, by improving the cognitive radio users to the transmitter and the receiver sensitivity, on the other hand also need to use digital signal processing (DSP) with different definitions of network operators to gain, so as to achieve a higher, faster, more accurate spectrum detection ability. In view of the situation of low utilization rate of Spectrum, a research scholar in the field of communication according to the various network to access the time requirements, frequency band are differences in the requirements and space requirements, puts forward the Advanced Spectrum Management (Advanced Spectrum Management, ASM) technology, in order to realize the frequency Spectrum of the system open, so as to reasonably allocate free wireless Spectrum resources. This not only can provide mobile users with better service quality, still can bring more benefits for the network operators. The advanced spectrum management can be divided into two categories: dynamic spectrum sharing technology and dynamic spectrum access technology.

As one of the key technologies of cognitive radio, the Dynamic Spectrum Access (Dynamic Spectrum Access, DSA) technology is focused on how to allocate channel, how to control the transmission power, how to avoid the interference and how to scheduling, thus seeking the reasonable use of idle Spectrum Access strategy. DSA technology can be summarized as dynamic exclusive use, open sharing and hierarchical access these three models.

Dynamic exclusive use 1 model [8]

Dynamic exclusive use of the basic structure of the model the same as the static Spectrum Allocation policy, mainly including Spectrum Property (Spectrum Property Rights) and Dynamic Spectrum Allocation (Dynamic Spectrum Allocation) of the two technologies. Among them, the use of the frequency spectrum property need to advance to a spectrum of rights shall be clear limits and define all aspects, such as the use of time, the use of geographical scope, communication signal power and signal transmission spectrum, etc., [9]. Obviously, these entities as strict execution, on the realization of technology have a great deal of difficulty.

Dynamic spectrum allocation is a kind of on-demand distribution of spectrum allocation methods, mainly used in the field of business to solve the heterogeneous spectrum allocation problem in cellular networks. At present, the research results are basically focused on single operator scene dynamic spectrum management. But many operators scene is a very promising in the future wireless communications a dynamic spectrum management method [10].

In the wireless communication network, each user's transmission power is more or less can bring interference of the surrounding the user's communication, to reduce such interference in the system, at the same time maximize the system capacity, power control technology for shipment. For CR system, it is essential to one of the key technologies. In order to enlarge the scope of work of the communication system, distributed on the CR users transmitter power control, which can ensure the power emitted by each CR users in the network in minimize, arrived at the base station can meet the basic communication requirements. But in a multi-user transmission of CR system, sending power control by the interference of a given temperature and the limitation of available spectrum whole number, at present to solve the problem of power control is the main technology of the game theory and information theory.

\section{Dynamic Spectrum Access Strategy Design}

Assumption in A network of CR users A and B CR users need to communicate, CR users C is CR A or B CR users A neighboring user DCA strategy channel model is the 
single free spectrum into public control channel and data channel, and CCA - MAC strategy is to assume that there is A specific spectrum as A common control channel, labeled $\mathrm{CHO}$; All data channel is through the physical layer of CR technology to detect one or more of the idle spectrum, labeled $\mathrm{CH} 1$ and $\mathrm{CH} 2, \ldots, \mathrm{CHN}$. Each CR users in the network have on the basis of the data channel cognition, first is provided by the physical layer and updated in real time, such as the main user channel utilization rate, average packet size and channel state information such as the list. Among them, the main user channel utilization rate, the greater the means that the user interrupt CR users in the letter on the greater the probability of data transmission. Next time slot of the main user channel utilization rate and the average packet size can be calculated by the relevant data of the current time slot. In addition, the channel state information in the list by $\mathrm{CR}$ users on the public control channel $\mathrm{CHO}$ updated control group interaction. On the public control channel $\mathrm{CH} 0$, CR users to send their own set of available channel, RTS packet may be the information such as the transmission time, this is similar to the IEEE 802.11 Distributed coordination Function (Distributed Coordinated Function, DCF model, and by receiving the other $\mathrm{CR}$ to record and update user information. The state of data channel each data channel available time is based on the main user channel utilization and probability theory to forecast, $\mathrm{CR}$ users tend to use low main channel utilization channel for data transmission. Therefore, we need to communication of CR user on the receiving user will reply the CTS packet to send the user; the CTS packet contains a data channel information such as the longest time available. Then, launch users to send confirmation RTS (Confirm - RTS, CRTS) packet to its adjacent to the user, to alert the upcoming communication. As the main users wishing to communicate with the $\mathrm{CR}$ users to use the same channel at the same time, the main users have absolute priority.

CRAHN topology structure is often used in some special occasions, such as military, medical, etc. For these occasions, reduce the link between the interference spectrum utility is more important than maximum link. So, on the basis of the dynamic transmission power control, in order to further reduce the interference between the CR links, this paper designed a kind of access selection mechanism. In this mechanism, in view of the uncertainty of CR link interference value, using the probability distribution of the interference value, the threshold for adaptive interference Ith the calculation of available and interference value corresponding information entropy $\mathrm{H}$ to estimate a probability distribution. When a CR link to other CR link interference than Ith, does not allow the link to access free spectrum for communication, on the other hand, can access the free spectrum for communication. Access to the screening mechanism, specific as follows:

Step 1, calculate CR link I always interference in other CR link Ii

$$
I_{i}=p_{i} \sum_{j \neq i}^{M} h_{i j}
$$

Step 2, calculating Ith: suppose the possible interference threshold for $\mathrm{m}$, a for Ii minimum, maximum of $\mathrm{b}$ said $\mathrm{Ii}$, the interference power value is less than the probability of $\mathrm{mP} 1$ and interference power values greater than or equal to the probability of $\mathrm{m} \mathrm{P} 2$ as shown on the type (2) :

$$
\left\{\begin{array}{c}
P_{1}=P(\mathrm{~m})=\sum_{I_{i}=a}^{m} P_{I_{i}} \\
P_{2}=1-P(\mathrm{~m})
\end{array}\right.
$$

Among them, $P_{I_{i}}$ for when the CR link to other CR Ii link the probability of interference is Ii, so accordingly with $\mathrm{P} 1$ and $\mathrm{P} 2$ information entropy are: 


$$
\left\{\begin{array}{c}
H(1)=\log _{2} P(\mathrm{~m})+H(m) / P(m) \\
H(2)=\log _{2}[1-P(\mathrm{~m})]+[H(b)-H(m)] /[1-P(m)]
\end{array}\right.
$$

where

$$
\begin{aligned}
& H(m)=-\sum_{I_{i}=a}^{m} P_{I_{i}} \log _{2} P_{I_{i}} \\
& H(b)=-\sum_{I_{i}=m}^{b} P_{I_{i}} \log _{2} P_{I_{i}}
\end{aligned}
$$

The total entropy $\mathrm{H}=\mathrm{H}(1)+\mathrm{H}(2)$, select interference threshold $\mathrm{Ith}$ is makes the biggest $\mathrm{m} \mathrm{H}$ value.

Step 3, set up access screening matrix A:

If Ii Ith, or stop CR link I access available spectrum, and A (I) = 0; If Ii < Ith, allowing the CR link I access available spectrum, and

$\mathrm{A}(\mathrm{I})=1$.

Step 4, ends with the unceasing change of network structure, CR link based on dynamic threshold access interference spectrum.

\section{Simulation Results and Analysis}

Do not break in general, considered within the scope of architectural CRAHN topology. CR link random evenly distributed in the range. The simulation spectrum bandwidth choice $\mathrm{B}=128 \mathrm{hz}$; CR link power loss index of $\alpha=4 ; P_{i}^{\max } / n_{0}=40 \mathrm{~dB}$; Each of the transmitted power in the range of CR link $p_{i} \in\left[p_{i}^{{ }^{\mathrm{min}}}, p_{i}^{\mathrm{max}^{\mathrm{ax}}}\right]=[0.0001 w, 1 w]$; Contain precision $\varepsilon=0.0001$. Do not break in general, the odd number of CR link power dynamically.

The Figure 1 shows that when the possible interference threshold $\mathrm{m}=0.15 \mathrm{mw}$, the maximum information entropy, so the choice interference threshold $\mathrm{Ith}=0.15 \mathrm{mw}$. By type (3) (4) (5), the Ith value with the change of topological structure may be different.

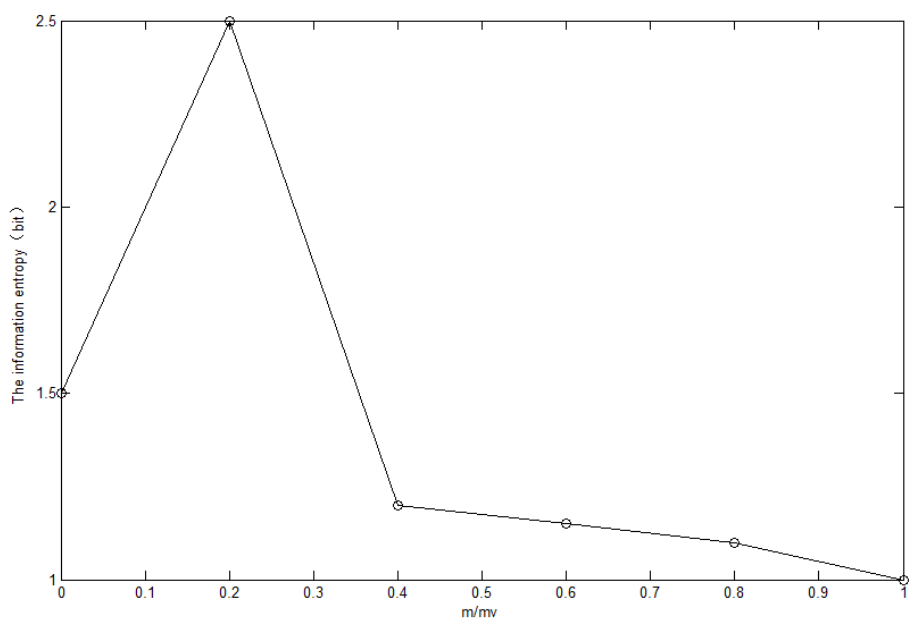

Figure 1. Interference Threshold Selection

In Figure 2, the curve of $\mathrm{x}$ said the proposed dynamic power control + screening threshold access control scheme of total CR link between interference, - said CR link using a fixed point curve transmission power, neither to transmit power under the condition of dynamic adjustment of CR link between total interference. Obviously, 
based on the dynamic transmission power control and the proposed access screening mechanism can largely reduce the total CR link between interference.

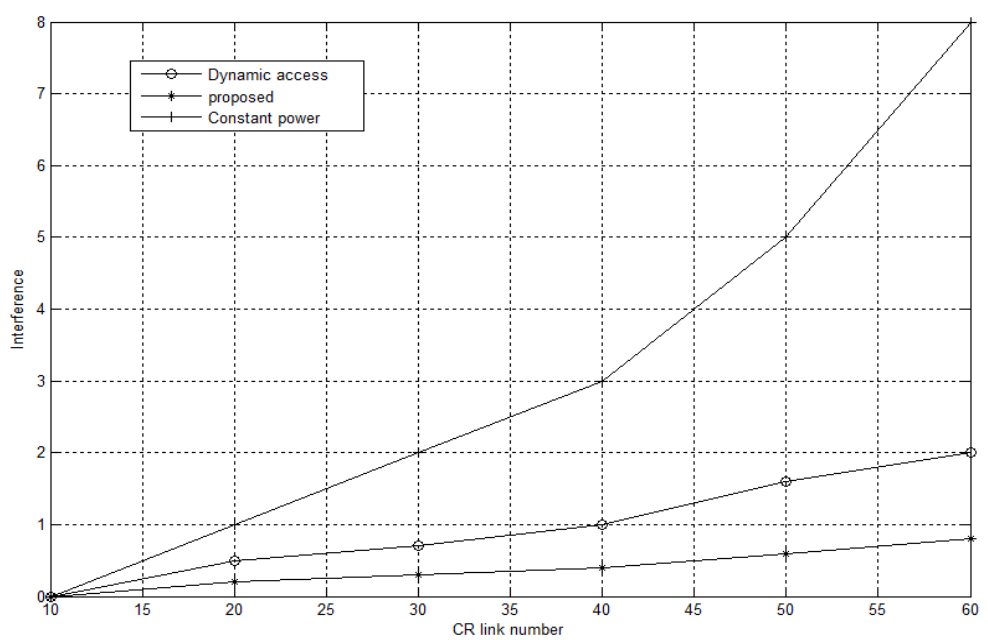

Figure 2. Interference Contrast Different Schemes

\section{Conclusion}

Of CRAHN DSA, the above presented a dynamic spectrum access based on transmission power control mechanisms. First by power spectrum utility cost and asynchronous contain algorithm to obtain the optimal power distribution, so as to maximize the spectrum utility of CR link. Then, add dynamic spectrum access mechanisms to reduce the total CR link between interference, the dynamic spectrum access threshold selection mechanism of the adaptive interference Ith through one-dimensional maximum entropy method. The simulation results show that the scheme in the aspect of reducing the amount of CR link between interference can achieve better performance. In addition, the proposed scheme can also be used for equipment of equipment (D2D) communication system, because the D2D communication scenarios and compact Ad Hoc structure is very similar.

\section{References}

[1] W. B. Heinzelman, A. P. Chandrakasan and H. Balakrishnan, "An application-specificprotocol architecture for wireless micro sensor networks", IEEE Trans, on Wireless Communications, vol. 1, (2002) October, pp. 660-670.

[2] S. Cui, A. J. Goldsmith and A. Bahai, "Energy-efficiency of MIMO and cooperative MIMO techniques in sensor networks", IEEE Journal on Selected Areas in Communications, vol. 22, (2004), pp. 1089-1098.

[3] S. K. Jayaweera, "Virtual MIMO-based Cooperative Communication for Energy-constrained Wireless Sensor Networks", IEEE Trans, on Wireless Communications, vol. 5, (2006), pp. 984-989.

[4] X. Li, M. Chen, and W. Liu, "Application of STBC-encoded cooperative transmissions in wireless sensor networks”, IEEE Signal Processing Letters, vol. 12, (2005), pp. 134-137.

[5] Y. Yuan, M. Chen and T. Kwon, "A novel cluster-based cooperative MIMO scheme for multi-hop wireless sensor networks”, EURASIP Journal on Wireless Communications and Networking, (2006), pp. 1-9.

[6] R. N. Paulraj and D. Gore, "Introduction to Space-Time Wireless Communications", Cambridge University Press, Cambridge, U.K., (2003).

[7] S. K. Jayaweera, "Energy analysis of MIMO techniques in wireless sensor networks", In Proceedings of the 38th Annual Conference on Information Sciences and Systems (CISS), (2004) March.

[8] S. K. Jayaweera, "An energy-efficient virtual MIMO Communications architecture based on V-BLAST processing for distributed wireless sensor networks", Sensor and Ad Hoc Communications and Networks, 2004 First Annual IEEE Communications Society Conference on, (2004), pp. 299-308.

[9] M. Dohler, E. Lefranc, and H. Aghvami, "Virtual antenna arrays for future wireless mobile communication systems, ICT 2002, Beijing, China, (2002) June.

[10]M. Dohler, E. Lefranc, and H. Aghvami, "Space-time block codes for virtual antenna arrays" PIMRC, Lisbon, Portugal, (2002) September. 


\section{Authors}

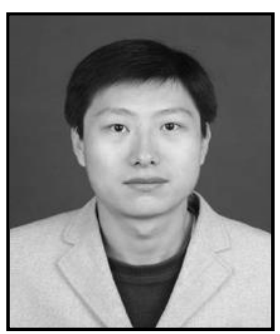

Zhipeng Song, he received the master's degree in engineering in Computer Software and Theory from Shandong Normal University in 2004.His current research interests on Computer Network Security and Computer application. 
International Journal of Future Generation Communication and Networking Vol. 8, No. 2 (2015) 